\section{Image Gallery: Acquired zinc deficiency of prematurity}

DOI: $10.1111 /$ bjd. 16142

DeAr Editor, An extremely premature male infant was discharged home after 11 weeks of intensive care. After 1 week, brownish-red, eroded macules and papules developed on the face, and also brown macules, plaques and bullae appeared on his fingers, hands, wrists, ankles, feet and toes. Despite multiple antibacterial and anti-inflammatory treatments administered to the patient as the diagnosis was thought to be impetigo, a significant worsening of the symptoms was observed. Laboratory testing demonstrated an unusually low serum zinc level, which supported the diagnosis of acquired zinc deficiency of prematurity. The introduction of supplementation therapy resulted in a rapid improvement of the clinical symptoms within days. Classic acrodermatitis enteropathica was excluded by testing the SLC39A4 gene.

${ }^{1}$ Department of Dermatology and Allergology,

University of Szeged, Szeged, Hungary

Z.R. CSOMA ${ }^{1}$ (iD)

L. KEMÉNY ${ }^{1,2}$

${ }^{2}$ MTA-SZTE Dermatological Research Group,

Szeged, Hungary

E-mail: csoma.zsanett@med.u-szeged.hu

Funding sources: none.

Conflicts of interest: Z.R.C. is supported by the János Bolyai Research Scholarship from the Hungarian Academy of Sciences (2015-2018).

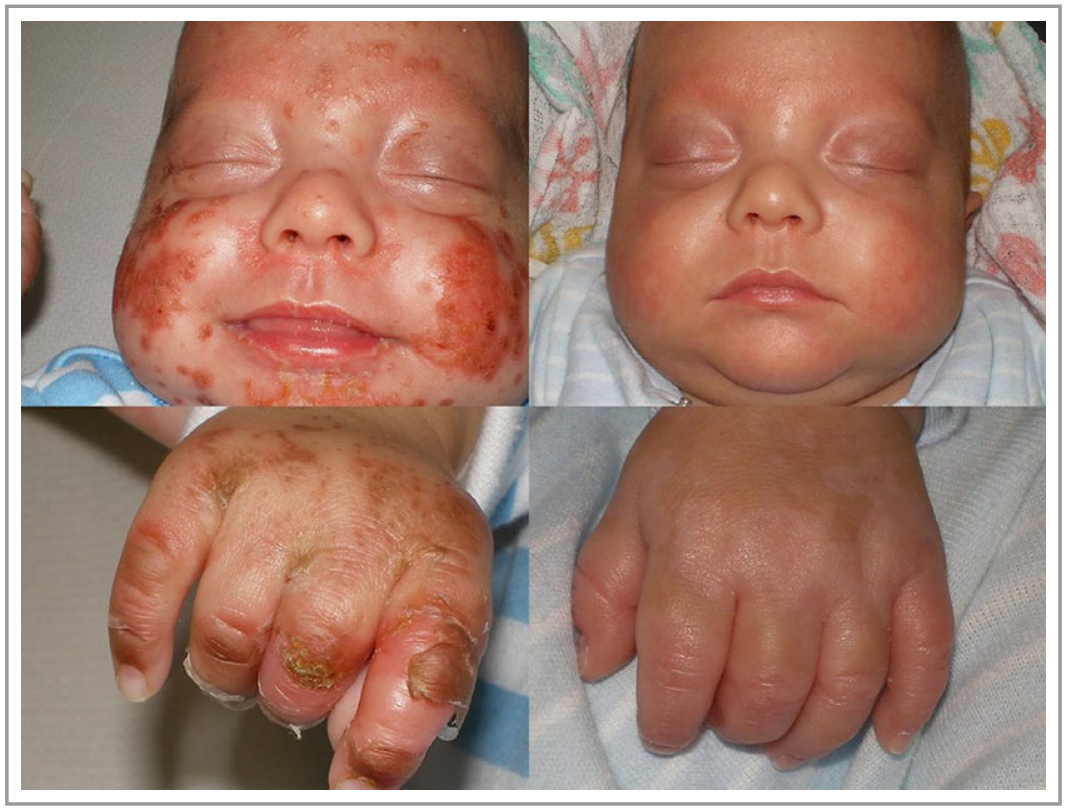

